\title{
L'homme, la technique et la vie dans la philosophie de Hans Jonas
}

Une approche critique

\section{Xavier Guchet}

\section{OpenEdition}

\section{Journals}

Édition électronique

URL : http://journals.openedition.org/alter/295

DOI : $10.4000 /$ alter.295

ISSN : 2558-7927

Éditeur :

Association ALTER, Archives Husserl (CNRS-UMR 8547)

Édition imprimée

Date de publication : 15 novembre 2014

Pagination : 79-99

ISBN : 978-2-9550449-0-2

ISSN : 1249-8947

\section{Référence électronique}

Xavier Guchet, "L'homme, la technique et la vie dans la philosophie de Hans Jonas », Alter [En ligne],

22 | 2014, mis en ligne le 01 décembre 2017, consulté le 19 avril 2019. URL : http://

journals.openedition.org/alter/295; DOI : 10.4000/alter.295

\section{Ce document a été généré automatiquement le 19 avril 2019}

Revue Alter 


\section{L'homme, la technique et la vie dans la philosophie de Hans Jonas}

Une approche critique

Xavier Guchet

Introduction

1 Dans ses Essais philosophiques ${ }^{1}$, Hans Jonas rattache son questionnement sur la technologie moderne à une perspective de philosophie morale. L'introduction du recueil est en effet pour lui l'occasion de revenir sur l'ensemble de son parcours intellectuel, et de préciser dans quel contexte son «attention philosophique » s'est portée sur le « défi moral de la technologie moderne $»^{2}$. Abandonnant l'optimisme un peu naif qu'il confesse avoir nourri à l'égard des possibilités de la technologie en matière de paix mondiale et de bonheur de l'humanité, Jonas explique que l'aggravation des problèmes écologiques ainsi que le développement des technologies génétiques l'ont amené dans les années 1970 à se fixer comme nouvelle tâche philosophique l'élaboration d'une éthique à la mesure de ces modalités inédites de l'agir humain. Pour autant, Jonas ne considère pas cette focalisation sur l'urgence d'une "éthique pour la civilisation technologique $»^{3}$ comme une franche rupture par rapport à ses problématiques antérieures. En particulier, il explique que son problème consiste à fonder cette éthique sur la philosophie de l'organisme telle qu'il l'a exposée dans son ouvrage Le phénomène de la vie $e^{4}$. En effet, l'éthique doit fournir de nouvelles normes pour l'agir humain, or ces normes trouvent leur fondement dans "l'image » de l'objet sur lequel porte cet agir - c'est-à-dire dans une «image de l'homme» (an image of Man), sur laquelle les technologies génétiques en plein essor donnent désormais une prise directe. À quelle «image de l'homme » faut-il se référer pour fonder les normes d'action dont nous avons cruellement besoin aujourd'hui ? Jonas a établi par ailleurs que cette "image de l'homme » ne peut être déterminée qu'à partir d'une philosophie de la vie qui l'englobe. Celle-ci est en effet à la fois une philosophie de l'organisme et une philosophie de l'esprit. L'anthropologie, qui cherche à rendre compte de cette «image de l'homme» et de la singularité de la situation humaine dans 
l'ensemble du monde vivant, est donc une partie de la philosophie de la vie. En résumé, la pensée de la technique de Jonas serait circonscrite au constat d'une transformation en profondeur des modes de l'agir humain - constat qui, comme on sait, ouvre Le principe responsabilité - et à l'exigence d'un renouveau de l'éthique face à cette situation inédite. La technologie moderne appelle une réflexion normative urgente. Ces fondements normatifs ne peuvent être trouvés que dans le cadre d'une anthropologie à même de ressaisir l'homme du point de vue de la vie et de son mouvement.

2 Peut-on en rester là cependant ? Peut-on limiter la pensée de la technique de Jonas à ce défi d'une éthique pour la modernité technologique? On entend montrer que non. La question de la technique reçoit chez Jonas un traitement philosophique qui ne se réduit pas au cadre posé par le premier chapitre du Principe responsabilité. Plus précisément, Jonas confère à la technique un statut philosophique qui déborde ce cadre de la réflexion éthique à l'ère des menaces écologiques et des manipulations génétiques. Pour le dire autrement, il y a chez Jonas une conception globale de la technique qui n'est pas commandée par l'opposition, trop abrupte, entre la technique traditionnelle et la technologie moderne - une philosophie plus générale de la technique, en somme, pardelà les caractéristiques à bien des égards effectivement inquiétantes des nouvelles technologies. On se propose dans un premier temps de revenir sur la façon dont Jonas met la technologie moderne au centre de ses préoccupations dans les années 1970, en en précisant les aspects nouveaux eu égard aux modalités classiques de l'agir humain. On examinera ensuite dans quelle mesure cette idée d'un renversement radical, d'une inversion pure et simple de signe entre technique traditionnelle et technologie moderne, doit être nuancée. On évaluera enfin la portée du questionnement sur la technique chez Jonas, au travers de ses réflexions sur la signification anthropologique de l'outil, en pointant les limites de ce questionnement dont les termes mêmes, il faut le souligner, se trouvent fragilisés par les connaissances accumulées en paléoanthropologie à l'époque où Jonas développe sa conception philosophique de l'outil, et qu'il ne semble pas prendre en considération. Cette «image de l'homme » que cherche Jonas peut-elle cependant faire l'économie d'une discussion avec ces connaissances scientifiques sur l'hominisation? Jonas ne sacrifie-t-il pas cette nécessaire discussion au maintien d'une "image» de l'homme que le préhistorien André Leroi-Gourhan ${ }^{5}$ qualifiait lui-même de «fantastique »? C'est ce qu'il convient d'examiner, en évaluant les distorsions entre, d'un côté, certaines assertions de Jonas touchant l'homme et son devenir, et d'un autre côté certaines connaissances en paléoanthropologie et en préhistoire solidement acquises dans les années 1970-1980, établies sur la base d'une documentation rigoureusement contrôlée.

\section{I - « Le défi moral de la technologie moderne »}

Jonas ne délimite pas précisément ce qu'il entend par « technologie moderne », celle-ci se signale d'abord par ses caractéristiques. Il en propose $\operatorname{six}^{6}$ :

4 -tandis que la technique relevait jusqu'à présent d'une évaluation de type anthropologique et instrumentaliste, c'est-à-dire selon la valeur morale des finalités et des usages (les techniques étant en elles-mêmes de simples moyens, moralement neutres, d'atteindre ces finalités), la technologie moderne se caractérise d'abord par le fait qu'elle intéresse la philosophie morale bien au-delà des intentions qui ont présidé à sa conception, à sa mise au point et à sa diffusion. Le «défi moral» de la technologie ne 
concerne plus seulement, ni même prioritairement, la question du bon ou du mauvais usage. En d'autres termes il n'est plus possible de soutenir que la technique est neutre en soi et que seuls relèvent du champ de la philosophie morale les usages que nous en faisons : au-delà des intentions, des buts visés et des usages, la technologie moderne pose des problèmes moraux inédits par le fait même que son déploiement a des effets indépendants des intentions initiales du technicien ou de l'usager. Des technologies utilisées selon les meilleures intentions du monde peuvent déclencher une chaîne indéfinie de conséquences conduisant à une véritable malédiction. La question n'est plus seulement celle du bien-fondé moral des applications, mais celle de l'agir humain sur la matière inerte et vivante, et des conséquences imprévisibles de cet agir ;

5 - l'agir technologique moderne s'est transformé en profondeur, de par la nature même de cet agir et plus précisément en raison des ordres de grandeur mis en jeu. Jonas illustre ce point en distinguant l'élevage et l'agriculture traditionnels d'un côté, et les biotechnologies de l'autre : tandis que dans le premier cas, l'intervention technique opère dans l'ordre de grandeur des caractéristiques phénotypiques des êtres (il s'agit d'améliorer ces caractéristiques par des croisements ou des hybridations habiles), les technologies modernes du vivant interviennent directement dans le plan de construction moléculaire des êtres. Sur ce point, Jonas distingue les vivants génétiquement modifiés destinés à servir de réservoirs de matières premières (on peut illustrer cela par les bactéries que l'on modifie génétiquement en vue d'en extraire des protéines produites en masse) et les êtres vivants génétiquement modifiés destinés à accomplir des tâches spécifiques directement dans la nature (par exemple des bactéries que l'on modifie génétiquement afin qu'elles dégradent les hydrocarbures en cas de marée noire). Jonas ne se limite donc pas à souligner le changement d'échelle des effets de l'agir technologique humain - sur la base du constat, souvent repris, que l'homme agit désormais comme une force géologique, c'est-à-dire comme une force de perturbation globale et irréversible des grands équilibres écosystémiques, et non plus seulement comme une force de perturbation locale et réversible comme cela a toujours été le cas : Jonas souligne aussi, plus profondément en un sens, que la transformation de l'agir humain porte surtout sur les modalités de cet agir, sur les échelles d'intervention et sur la nature des chaînes opératoires mises en jeu;

6 - une troisième caractéristique de la technologie moderne consiste dans le fait qu'il n'y a plus de distinction entre posséder un pouvoir d'agir et l'exercer, c'est-à-dire entre les deux sens aristotéliciens de la puissance. Jonas reprend ici une idée exprimée avec force surtout depuis les années 1950, à savoir que la technique est devenue une force autonome et que désormais, selon la loi dite de Gabor, tout ce qui est techniquement possible sera réalisé. Jacques Ellul avait déjà insisté lourdement sur ce point. Jonas précise toutefois le sens et la portée de la thèse autonomiste telle qu'il la conçoit: dans son acception classique, cette thèse ne signifie pas une autonomisation des réalités techniques ellesmêmes, mais une autonomisation du processus de leur création et de leur utilisation, devenues étrangères à d'autres considérations que celles portées par la logique du développement technologique. Or, diagnostique Jonas, cela a changé avec les techniques biogénétiques, du type manipulations des génomes: nous créons aujourd'hui de nouveaux êtres vivants qui sont destinés à venir prendre place dans le monde, et à y produire des effets que nous ne pouvons pas anticiper. Il y a donc urgence à rétablir les conditions d'une maîtrise de nos œuvres technologiques, plutôt que de continuer cette folle course en avant qui nous amène à nous laisser maîtriser par la technologie. La 
réflexion éthique ne peut plus se limiter à l'exercice du pouvoir d'agir, mais doit s'étendre à sa possession. Jonas milite ainsi en faveur d'un contrôle extra-technologique de la recherche (c'est-à-dire sur l'acquisition de la puissance, et non seulement sur son exercice), sur les modalités duquel il reste il est vrai assez elliptique. Qu'est-ce que cela peut signifier? Jonas évoque la possibilité d'une autorégulation de la science, c'est-à-dire l'idée d'une régulation des recherches par les scientifiques eux-mêmes, mais il écarte cette solution - peu crédible en effet à l'époque où Jonas écrit ces lignes, au début des années 1980, alors même que l'idée d'une régulation des recombinaisons d'ADN viraux et des manipulations génétiques en général par les généticiens eux-mêmes, réunis à la conférence d'Asilomar en 1975, n'a pas eu les résultats escomptés. Jonas évoque alors l'idée de commissions réunissant ce que l'on appelle aujourd'hui les parties prenantes, y compris les profanes, mais il n'y croyait pas dès lors que pour lui, ce type de commissions ne peut être que la caisse de résonance des intérêts de chacun, de chaque groupe de pression, et non de l'intérêt général. Il ne croyait pas davantage en l'idée d'un conseil des Sages, une sorte de comité de philosophes-rois à l'échelle mondiale. Jonas finit par botter en touche (ce qui en un sens peut lui être reproché) en concluant que ce problème de "gouvernance mondiale» de la science, comme on dit aujourd'hui, n'est pas son problème en sa qualité de philosophe préoccupé d'éthique et non de politique ${ }^{7} .$.

7 Les trois premières caractéristiques de la technologie moderne (les problèmes éthiques ne se limitent plus à l'évaluation des intentions, des buts visés et des usages; l'agir humain s'est transformé qualitativement ; il n'y a plus de distinction entre possession et exercice du pouvoir d'agir technologiquement) sont illustrées par Jonas au travers de la comparaison entre la bombe atomique et les technologies biomédicales. La bombe atomique est "mauvaise en soi », elle est indéfendable quant à ses finalités elles-mêmes ; les effets d'une explosion sont à grande échelle certes, mais la catastrophe est instantanée ; enfin, la distinction entre la possession et l'exercice du pouvoir n'est pas invalidée (fort heureusement, la catastrophe n'est pas inéluctable). La situation est très différente dans le cas des technologies biomédicales: les intentions sont parfaitement louables et même indiscutables dans la plupart des cas, en outre la conséquence est bonne de la possession à l'exercice du pouvoir (si l'on peut réduire la mortalité infantile dit Jonas en substance, on ne peut pas moralement ne pas le faire!) ; enfin, les effets de la mise en œuvre de ces technologies se font sentir sur le long terme. Si malédiction il y a, celle-ci ne se manifestera qu'avec retard;

8 - une quatrième caractéristique de la technologie moderne réside dans le changement d'échelle, à la fois spatiale et temporelle. Jonas constate tout d'abord un changement d'échelle dans la mise en œuvre des technologies, du côté du sujet : l'individu ne compte plus, c'est la société qui agit technologiquement. Il souligne également un changement d'échelle du côté du monde, dans l'ampleur des effets de l'agir technologique moderne (il suffit ici de renvoyer aux célèbres pages qui inaugurent Le principe responsabilité) ;

9 - en conséquence de ce changement d'échelle de l'agir humain, l'éthique ne peut plus selon Jonas être anthropocentrique, le point de vue utilitaire sur la nature doit être dépassé de façon à faire entrer la nature et la vie elles-mêmes dans le champ de la philosophie morale ;

10 - enfin, sixième et dernière caractéristique de la technologie moderne : elle nous oblige à poser la question métaphysique redoutable de savoir pourquoi il doit y avoir une humanité et même la vie en général. La technologie moderne est en effet une menace pour la vie elle-même, il est par conséquent urgent de justifier moralement les raisons 
pour lesquelles la vie doit être préservée. Si l'éthique dont nous avons besoin à l'ère de la technologie moderne doit être fondée dans la philosophie de la vie, c'est que la vie est menacée d'exténuation par la technologie moderne et que pour la défendre, il faut avoir préalablement justifié que la vie est en elle-même porteuse d'une valeur intrinsèque.

Ces développements sur la technologie moderne et sur la nécessité d'une éthique pour notre temps ont indéniablement constitué une clé de lecture privilégiée de la pensée de la technique de Jonas. Pour autant, ces analyses ne résument pas tout ce que Jonas dit de la technique. Il faut même avouer que sans autre précision, ces analyses pourraient laisser perplexe. En effet, Jonas parait vouloir ressaisir l'homme comme un vivant au destin particulier (l'anthropologie est une partie de la philosophie de la vie). L'outil est alors, on le verra, une manifestation de la vie quand elle se fait humaine ou encore, pour reprendre le terme de Jonas, «transanimale ». À travers l'outil, c'est donc encore la vie qui continue son mouvement. Or, brusquement la technologie moderne se retournerait contre la vie d'un point de vue totalement extérieur à elle, jusqu'à la menacer de disparition pure et simple. Comment comprendre un tel retournement au plan ontologique, sinon en situant cette tension de la vie et de la non-vie au sein de la vie elle-même? Les analyses de Jonas de la technologie moderne sont-elles compréhensibles si l'on ne fait pas du retournement que celle-ci opère contre la vie, une autonégation de la vie elle-même pour reprendre le mot de Renaud Barbaras? Pour comprendre le statut de la technologie moderne, il faut dès lors en trouver le fondement dans une tension opérant au sein même de la vie. Il faut plus précisément revenir au statut de la technique en général dans le mouvement de la vie, afin d'y repérer comment la technique comme telle, et non seulement la technologie moderne, est ce qui contribue à introduire dans la vie, et ceci du point de vue même de la vie et de son mouvement, le point de vue de la mort. Alors seulement les analyses de la technologie moderne pourront trouver leur pleine justification philosophique.

\section{II - La technique et la vie : une approche plus globale de la question}

Il convient par conséquent de nuancer et même de contester l'idée que chez Jonas, seule la technologie moderne serait problématique - la technique traditionnelle, celle fondée sur les outils, s'étant jusqu'alors déployée de façon absolument non problématique au service de la vie. Cette opposition est trop simpliste et ne traduit pas la pensée de Jonas, plus raffinée. Il apparaît en effet que pour Jonas, la technique est depuis toujours, et non seulement à l'époque contemporaine, un truchement de l'inquiétude, de l'expérience de la finitude et de la mort.

13 Ainsi, dans son ouvrage majeur ${ }^{8}$, Jonas appréhende-t-il la technique du point de vue de ce qu'il appelle "l'ontologie de la mort", sur laquelle la vie a été rabattue à l'époque moderne - notamment dans la science biologique qui veut à tout prix expliquer la vie par la non-vie. Jonas propose un récit très stimulant : la pensée des hommes des origines était moniste, panvitaliste au sens où la vie y était considérée comme le phénomène évident, massif, omniprésent, en un mot "naturel», tandis que la mort y était au contraire l'exception réclamant une explication. Cette posture non problématique à l'égard de la vie s'est inversée en une pensée pour laquelle la vie est une exception et un miracle en attente d'explication, l'inerte c'est-à-dire la non-vie étant au contraire le caractère normal des choses. « Notre pensée est aujourd'hui sous la domination ontologique de la 
mort $»^{9}$. Jonas situerait plus précisément dans l'avènement de la pensée et de la science dites modernes, au XVII ${ }^{\mathrm{e}}$ siècle, les racines philosophiques de cette technologie moderne si menaçante pour la vie. Cela étant, le monisme panvitaliste dont parle Jonas, bien loin d'être absolument non problématique, était déjà « inquiété (...) par l'expérience de la mort et graduellement érodé par la technique $\aleph^{10}$. Jonas est radical et somme toute conséquent : ce ne sont pas seulement les technosciences contemporaines qui sont exclues de la vie et opèrent sur elle d'un point de vue extérieur à elle, par un privilège qu'il serait après tout bien difficile de justifier au plan ontologique. Toute technique a un rapport extérieur à la vie et vient " l'inquiéter », mais il faut préciser que cette inquiétude se déploie au sein même de la vie, c'est la vie elle-même qui est autonégation - la technique est une altération de la vie du dedans même de la vie, si l'on peut dire. En effet, la vie est constitutivement inquiétude, épreuve du danger et de la mort.

Il est ici nécessaire de rappeler les célèbres analyses du Phénomène de la vie concernant le métabolisme, à partir du concept de médiation. Lorsque Jonas introduit la notion de métabolisme comme moment inaugural de la vie, comme forme première et support de son déploiement, il soutient que le végétal est finalement le vivant parfait, l'incarnation la plus pure de l'essence de la vie comme relation du vivant à ce qui n'est pas lui. La plante est en effet un vivant en relation immédiate avec un milieu contigu, dans lequel elle prélève les substances inorganiques dont elle a besoin pour fabriquer de l'organique. L'animal quant à lui est moins parfait, il ne peut pas fabriquer directement de l'organique à partir du minéral (à l'exception toutefois de certaines bactéries, qui connaissent une photosynthèse plus simple que celle des plantes), il s'inscrit dans une chaîne alimentaire dans laquelle les vivants ne peuvent se procurer les constituants inorganiques dont ils ont besoin (sels minéraux etc.) qu'en assimilant d'autres vivants, et ainsi de suite jusqu'au végétal. Entre l'animal et son milieu, une distance s'introduit qui l'oblige à devenir un être des médiations, un vivant voué au détour. En tant que médiation d'un genre somme toute particulier, la technique est un nouveau moyen au service du détour, et elle manifeste en outre un métabolisme dégradé par rapport à celui des plantes, un défaut dans la vie - défaut qui ouvre certes des perspectives nouvelles au vivant désormais mobile, mais défaut tout de même. La technique est par conséquent la forme proprement humaine que prend cette inquiétude, cette expérience de la finitude et de la mort, au sein même d'une vie «transanimale » certes, mais toujours vouée au détour et à la médiation. L'homme est le vivant en lequel cette autonégation de la vie accède à la conscience de soi, or la technique joue ici un rôle décisif. Jonas précise que le phénomène du cadavre est de même nature que celui de l'outil : tous deux viennent contrarier le monisme panvitaliste non problématique et ont puissamment contribué à ce revirement majeur dans la pensée humaine, en la faisant donc passer sous la domination d'une "ontologie de la mort»: «Tout comme la pratique de l'homme des premiers temps est incarnée dans ses outils, de même sa pensée est incarnée dans ses tombes qui à la fois reconnaissent et nient la mort $»^{11}$.

15 À ce point, la pensée de Jonas se complique. Une traduction importante de «l'ontologie de la mort » dans la pensée de la vie a consisté en effet à rabattre la vie sur la survie, à définir la vie comme ce qui doit constamment lutter contre les forces de mort afin de se préserver et de se perpétuer. L'on glisse ainsi subrepticement d'une notion de vie comme autonégation, c'est-à-dire d'une notion de vie comprise comme mouvement d'autoaltération, à une compréhension de la vie comme survie. De la vie comme épreuve de la finitude et de la mort dans le travail de la médiation, à la vie comme ensemble des forces 
qui résistent à la mort : une sorte d'inversion de valeur s'est opérée. Ainsi réduite aux conduites de la survie, la vie est irrémédiablement manquée dans son essence selon Jonas. Or, selon Jonas toujours, la technique a précisément fini par être considérée dans la seule perspective de la survie, comme un ensemble de moyens en vue de satisfaire des besoins nécessaires - selon cette perspective anthropologique et instrumentaliste à laquelle ne souscrit plus la technologie moderne, on l'a vu: mais on voit maintenant que cette perspective est déjà la conséquence d'un renversement de valeur affectant l'idée de vie. Bien loin d'être un obstacle à la compréhension de « l'essence de la technique » moderne, comme le voulait Heidegger, la perspective anthropologique et instrumentaliste, tributaire d'une conception de la vie comme survie, prépare ainsi l'idée que la technique est une force étrangère à la vie elle-même. Dès lors que Jonas cherche à ressaisir le phénomène de la vie pour lui-même, en son essence propre, c'est-à-dire autrement qu'en le rabattant sur ce qui n'est pas lui, il doit par conséquent inévitablement en exclure la technique ainsi comprise - ce que l'on voit bien dans Le phénomène de la vie, où la question de la technique apparaît somme toute relativement sous-déterminée. En particulier, il n'en est aucunement question lorsque Jonas récapitule les principaux acquis de sa philosophie de l'organisme, en guise de transition vers son anthropologie : les quelques pages qui introduisent aux chapitres sur la situation humaine dans le monde ne font aucune mention de la technique, ce qui peut sembler surprenant. Cela se comprend dès lors que la technique, effectivement essentielle dans le processus par lequel la vie s'est faite vie humaine, se trouve finalement déqualifiée en même temps que la vie est déqualifiée comme survie. En son essence la vie n'est pas la survie, elle est certes un rapport à ce qui n'est pas elle, une relation ininterrompue au non-soi, une extériorisation continuée ; le métabolisme est la manifestation universelle de cela : par son métabolisme, toute forme de vie (y compris les plus simples) est déjà ouverture au monde. Ne pourraiton pas alors soutenir que la technique, qui est elle aussi une forme et un support des relations que certains vivants ont à leur milieu, ne fait que spécifier, plutôt que contrarier, cette caractérisation universelle du métabolisme? Non, répond en substance Jonas : le métabolisme apporte effectivement la dimension de l'extériorité au vivant, mais il s'agit d'une extériorité qui définit la vie en son essence ; une extériorité qui opère par conséquent du dedans de la vie. La technique apporte une dimension d'extériorité tout à fait différente : non pas extériorité de la vie et dans la vie, mais extériorité par rapport à la vie - extériorité du dehors de la vie, qui s'impose à la vie comme ce qui est parfaitement étranger à son mouvement propre. La technique est tout au plus un adjuvant de la vie, elle désigne certaines des médiations par lesquelles certains vivants satisfont leurs besoins vitaux. Lorsqu'elle cesse d'être cet auxiliaire externe, et au fond inessentiel, de la vie, la technique finit par se retourner contre la vie et la menacer du dehors, d'un point de vue extérieur à elle - c'est exactement ce que Jonas pensait des technosciences contemporaines.

En résumé, le récit que propose Jonas dans Le phénomène de la vie concernant «l'ontologie de la mort " qui a fini par battre en brèche le panvitalisme des origines de la pensée humaine consiste à rendre compte d'une double déqualification : déqualification de la vie rabattue sur la survie, et déqualification de la technique rabattue sur la notion de moyen au service de la survie. Comme on sait, Jonas propose une réévaluation de notre concept de vie. À partir des concepts de métabolisme et de médiation, il entend retrouver un sens de la vie qui n'est pas la survie. Or, il semble que Jonas n'ait pas symétriquement réévalué le concept de technique. Son appréciation de la technologie moderne s'appuie sur une philosophie de la vie qui n'est effectivement plus rabattue sur la survie - ce qui lui permet 
de trouver des valeurs intrinsèques dans la vie; en revanche, le concept de technique semble demeurer inchangé, toujours tributaire de cette déqualification principielle. Si la technologie moderne n'est interrogée que sous l'angle de sa menace pour la vie, pour les coups qu'elle porte à "l'image de l'homme ", c'est que son concept reste tributaire du concept de la vie comme survie - ou, pour le dire autrement, c'est que la technique semble être désormais irrémédiablement coupée de la vie, purement extérieure à elle et à son mouvement propre. L'approche anthropologique et instrumentaliste est certes devenue intenable à l'ère des effets systémiques de la technologie et des conséquences imprévisibles de l'action humaine ; toutefois, le postulat au fondement de cette approche anthropologique et instrumentaliste demeure intact et continue de sous-tendre le concept de technique de Jonas : la technique est le moyen de la survie. Si la technologie moderne peut se retourner aussi brutalement contre la vie, d'un point de vue totalement extérieur à la vie, c'est que le concept de technique a déjà été exclu du concept de vie.

Il est vrai qu'il arrive à Jonas, et il faut le saluer sur ce point, d'ouvrir des pistes de réflexion originales concernant la technologie moderne, irréductibles au thème d'une technique se retournant purement et simplement contre la vie. Jonas se livre ainsi à un examen de la technologie moderne dans une perspective qui n'est pas seulement celle de la menace et de la catastrophe. Une illustration emblématique de cet élargissement du questionnement se trouve dans son discours de remerciement à l'occasion de la remise du prix de la Paix des éditeurs allemands, en octobre 1987 :

la technique prémoderne tout entière était macroscopique, comme l'étaient également les instruments anciens ou comme l'est aujourd'hui encore la machine. En manipulant des grandeurs relatives au monde corporel visible, la technique se tenait encore pour ainsi dire à la périphérie des choses. Depuis, elle a pénétré jusqu'au niveau moléculaire : elle peut désormais le manipuler, et créer à partir de là une matière qui n'a jamais existé, modifier les formes de la vie, libérer des forces. Jamais auparavant, on n'a serré d'aussi près le génie de la nature dans ses composantes. C'est à partir des tréfonds de la nature que la technique gouverne le niveau supérieur et, à partir de l'infinitésimal, qu'elle gouverne ce qui est immense 12 .

18 Jonas a en outre livré une analyse intéressante qui peut éclairer le sens de cet exposé13. L'affection des sens, explique-t-il, se produit à une «échelle minuscule par rapport à l'organisme ", c'est-à-dire à l'échelle nanométrique. Les sens sont affectés par des chocs, des heurts, des pressions à l'échelle moléculaire, bref le sentir repose sur des interactions physiques réelles, impliquant de nombreuses entités à très petite échelle. Les organismes vivants qui se situent dans cet ordre de grandeur n'ont affaire qu'à ces interactions moléculaires, lesquelles ne font pas encore une perception du monde. Pour que celle-ci puisse se constituer, il faut que ces innombrables interactions moléculaires soient en quelque sorte « moyennées », c'est-à-dire intégrées dans une configuration d'ensemble où elles n'apparaissent plus comme chocs élémentaires. La perception naît d'une image qui résulte elle-même d'une somme d'interactions infinitésimales. Ainsi, comme dit en substance Jonas, pour l'organisme de grande échelle, l'expérience du monde perçu "survient toujours déjà superposée au continuum apaisé constitué des transcriptions perceptives des influences de petite échelle qui tout au long engagent la sensibilité, mais perdent dans la transcription le caractère d'influence $»^{14}$. Or, cette opération de transcription, ou de moyenne, décide de la fonction objectivante des sens. Plus la réalité dynamique des interactions moléculaires de l'affection sensorielle est neutralisée, plus cette réalité est transcrite en perception d'une présence statique superposée à ces interactions, et plus le sens ouvre sur un monde d'objets. Jonas constate de ce point de 
vue le privilège de la vision : "d'une analyse comparative, la vue ressort comme le sens doté de la plus complète neutralisation du contenu dynamique et de la moins ambiguë des mises à distance de son objet par rapport à la fonction perceptive $»^{15}$. Une dernière précision: pour Jonas, la construction du perçu par intégration des interactions élémentaires dans un continuum « apaisé » implique que l'organisme de grande échelle, percevant un monde, a perdu le sens véritable de la force - il n'a accès qu'à la présence contenue d'un substrat statique, donné là-devant sans effort, vidé de toute référence aux dynamiques moléculaires sous-jacentes. L'expérience de la force est comme surajoutée par l'organisme de grande échelle, après coup, à un monde perçu qui s'est construit précisément en faisant disparaître dans la «transcription » perceptive la réalité des forces à l'œuvre au niveau élémentaire.

Ce développement est fort intéressant eu égard aux développements contemporains de technologies que Jonas ne connaissait pas, puisqu'il s'agit des nanotechnologies, en plein essor depuis la fin des années 1980 et liées à la mise au point de nouveaux instruments permettant à la fois d'agir sur des objets moléculaires individuels et de les imager : les microscopes en champ proche.

Les nanotechnologies semblent en effet renverser du tout au tout l'analyse de Jonas, et témoignent ainsi de sa capacité à faire comprendre la signification et la portée épistémiques des transformations en cours dans les technosciences. En effet, un nanoobjet ne gagne pas son objectivité par une sorte de lissage ou d'occultation des interactions moléculaires dont il est le siège ; au contraire, c'est dans la description fine et la compréhension de ces interactions (au point de vue notamment de ses relations de surface) qu'il acquiert sa consistance d'objet pour le savoir, et de support pour l'action technique. Imager le nano-objet ne signifie pas passer au-dessus les interactions effectives qui s'établissent entre lui et l'instrument, pour en fournir une perception statique et moyennée : imager le nano-objet signifie à l'inverse acquérir des données de manipulation sur eux, l'image conserve ici l'aspect dynamique qui se trouve justement évacué dans la perception au sens courant du terme, si l'on suit Jonas. Imager un nanoobjet ne veut pas dire construire une représentation statique d'un objet: cela veut dire imager une action sur un nano-objet, une manipulation dynamique qui opère au moyen d'un microscope en champ proche. En solidarisant étroitement la définition des nanoobjets et les relations dynamiques dans lesquelles ils sont pris, et qui les constituent comme objets, les nanotechnologies nous introduisent par conséquent à une situation tout à fait inédite si l'on se réfère à la grille d'analyse de Jonas : des organismes de grande échelle - nous -, qui ont développé les sciences de la nature, c'est-à-dire un savoir qui repose précisément sur le primat théorétique de la vision et sur une définition statique de l'objet, vidé de toute référence à l'origine dynamique de son contenu - ces organismes que nous sommes constituent désormais leurs objets de connaissance et d'intervention technique de telle façon que leur contenu dynamique ne soit justement plus du tout « neutralisé ». Autrement dit, imager un nano-objet n'est pas fournir une représentation coupée des forces qui s'exercent, mais au contraire fournir des données quantitatives concernant ces forces.

21 Au-delà du thème de la menace pour la vie, la lecture de Jonas permet ainsi de pointer des aspects fort intéressants liés au développement des nouveaux modes de confrontation à la matière - transformation dans la façon de se rapporter perceptivement au réel, de fabriquer des connaissances etc. Malheureusement, Jonas n'a pas développé plus avant ces intuitions. Il semble finalement en rester au constat que la capacité d'agir 
technologiquement sur la matière dans des ordres de grandeur jusque-là impraticables, doit avant tout être interrogée pour le type de dangers nouveaux et extrêmes auxquels elle nous introduit. Nul doute que cet aspect est essentiel, toutefois il y a une autre réalité de la technique dont Jonas semble avoir écarté le concept : une réalité dans laquelle la technologie moderne continue de manifester la capacité humaine d'inventer de nouveaux modes de confrontation à la matière, de nouvelles façons de se fabriquer des territoires et de percevoir le monde, à l'instar de ce que tout vivant est amené à faire. La technologie moderne continuerait-elle aussi l'aventure de la vie, dans toute son ambivalence il est vrai.

Il y a bien des domaines du savoir qui auraient pu permettre à Jonas de requalifier le concept de technique en même temps que le concept de vie, c'est-à-dire de ressaisir la technique autrement que sous l'angle de la survie - plutôt sous l'angle du mouvement de la vie elle-même : pensons en particulier à l'éthologie et à la paléoanthropologie. Or, si Jonas ne semble pas s'occuper d'éthologie, il aborde ouvertement des questions de paléoanthropologie lorsqu'il thématise l'homme comme «transanimal». Les paléoanthropologues ont en effet été amenés à ressaisir la technique autrement que comme un moyen de la survie, plutôt comme un fait biologique ayant une signification majeure dans le processus par lequel la vie s'est faite vie humaine. Qu'est-ce que Jonas tire de ces enseignements? Force est de constater qu'il n'en a pas tiré argument pour réviser son concept de technique, en ressaisissant celle-ci, y compris dans ses manifestations les plus contemporaines, dans la perspective du mouvement même de la vie et de son autonégation. Il convient pour finir d'examiner ce rendez-vous raté.

\section{III - Une critique de la conception jonassienne de la technique d'un point de vue paléoanthropologique}

Jonas ne paraît trouver dans l'éthologie aucun éclairage utile pour sa propre pensée de la technique. Il n'aborde pas la question des techniques animales, pour lui la technique est le propre de l'homme - en tout cas la technique chez l'homme est tout à fait spéciale, et il n'y a pas lieu de faire un détour par l'animal pour saisir la signification et la portée de la technique humaine. La technique nous installe d'emblée dans l'humanité. Il faut ici admettre que Jonas eût été bien inspiré de s'intéresser d'un peu plus près à l'éthologie, cela lui aurait évité des formules du genre: «Aucun animal n'ensevelit ses morts ou même ne continue à leur prêter attention ${ }^{16}$, ce qui est parfaitement inexact.

En revanche, Jonas a jugé important de remonter aux témoignages des hommes préhistoriques pour étayer sa conception de l'homme et pour préciser le statut de la technique dans l'humanisation. Cette confrontation au domaine de la paléoanthropologie se trouve particulièrement développée dans le texte "Outil, image et tombeau. Du transanimal dans l'humain $»^{17}$.

Jonas se fixe la tâche d'une anthropologie philosophique dont les réquisits sont indiscutables : essayer de rendre compte de la différence anthropologique, c'est-à-dire de ce qu'il y a d'entièrement original chez l'homme, sans pour autant couper l'homme du monde vivant. Il s'agit ainsi de "réfléchir à ce qu'il y a d'essentiellement transanimal dans l'homme, sans pour autant nier ce qu'il y a en lui d'animal. Au contraire, ce qui là dépasse toute animalité, nous pouvons justement le comprendre comme un nouveau degré de la médiateté dans la relation au monde qui se constitue au sein de l'existence 
animale, médiateté qui se superpose déjà au médié de toute existence organique en tant que telle, et sur laquelle s'érige la nouvelle médiateté, accrue, dans l'humaine relation entre le monde et le soi - mais comme une nouveauté d'essence, et non simplement de degré ${ }^{18}$. Il faut reconnaître que la formulation du problème est très précise et, redisonsle, indiscutable au regard des exigences auxquelles prétend souscrire une anthropologie philosophique aujourd'hui. En effet, sauf à vouloir livrer une «image fantastique » de l'homme, selon le mot déjà cité du paléoanthropologue et préhistorien Leroi-Gourhan, une anthropologie doit commencer par ressaisir l'homme selon les mêmes catégories d'analyse que les autres vivants, en essayant de qualifier la différence anthropologique sur fond de cette commune appartenance à la vie. Sur ce point, la démarche de Jonas est totalement conforme aux critères d'une approche scientifique de l'hominisation, objet de la paléoanthropologie. Il convient par conséquent d'examiner plus en détail le traitement que Jonas fait des savoirs paléoanthropologiques dont il pouvait prendre connaissance.

L'entrée en matière de Jonas sur ce point, dans le texte «Outil, image et tombeau. Du transanimal dans l'humain », surprend d'emblée : il explique en effet que sa méthode « va consister à sélectionner quelques signes caractéristiques de l'humain pour en interroger le sens ». Curieuse démarche en vérité : Jonas "opte» (selon son expression toujours) pour certains témoignages qu'on ne saurait attendre d'un animal, en l'occurrence l'outil, l'image, la sépulture («Mon choix s'est porté sur l'outil, l'image et le tombeau»). Il semble ainsi suggérer, d'une part que ces trois items sont des manifestations parmi d'autres de l'hominisation, d'autre part qu'il s'agit d'une affaire de choix personnel, d'option parmi une gamme de choix possibles. L'impression d'arbitraire qui se dégage de ces formules peut étonner, alors même que ces matières ont justement, fort heureusement, été arrachées à l'arbitraire pour devenir des objets de science. Quoi qu'il en soit, Jonas commence donc par l'outil, lequel «relève déjà de l'homme du fait précisément qu'il n'a rien en soi à voir avec l'homme, ne découle d'aucune fonction organique et n'est soumis à aucune programmation biologique $»^{19}$. Nous sommes clairement ici aux antipodes d'une philosophie biologique des techniques, c'est-à-dire de l'idée d'un ancrage vital des techniques. L'outil est dans la dépendance d'une faculté mentale, l'imagination, qui fournit les notions de forme et de matière - la représentation d'une forme appliquée à une matière. Cela étant, l'outil reste au plus près des fins vitales de l'animal. L'outil se tient dans la plus étroite relation avec la nécessité animale, elle sert les besoins de cette nécessité sur le mode du transanimal - bref, il permet à un être qui n'est plus simplement un être-en-vie d'assurer ses besoins vitaux. En ce sens, l'outil situe l'homme dans une simple différence de degré par rapport à l'animal, tandis que l'image et la sépulture introduisent une rupture d'essence. Statut paradoxal de la technique donc, et ceci dès l'outil : celui-ci est en effet au service de la survie, des buts vitaux, mais il apparait chez un être dont la vie est justement autre chose que la survie. L'outil se rencontre chez un être dont les caractéristiques essentielles ne se retrouvent pas chez les autres vivants, et ne trouvent pas non plus leur origine dans la vie elle-même ; pourtant, l'outil reste ce qui, chez l'homme, est très près de la vie comprise comme survie.

L'outil se tient donc " au plus près des fins vitales de l'animal ", mais il engage l'homme dans une aventure qui n'est déjà plus l'aventure de l'être-en-vie. Jonas situe précisément cette différence d'essence : l'outil répond à des stéréotypes, il est transmissible, en outre l'homme est capable de fabriquer des outils à fabriquer des outils. Enfin, on vient de le dire, l'outil humain est dans la dépendance de la faculté d'imaginer et de former des images, laquelle fait entrer l'homme dans le temps de l'invention ininterrompue. On ne 
peut pas ici ne pas penser à Bergson et à sa thématisation de la technique humaine dans la double référence à la faculté de fabriquer des outils à fabriquer des outils d'une part, et à la faculté fabulatrice d'autre part. Jonas ressaisit plus exactement l'outil sur le registre de l'imagination ludique, ce qui est intéressant puisque cela signifie que l'outil ne se trouve pas rabattu sur la seule utilité. De ces quelques lignes, il ressort par conséquent que l'outil a un statut ambivalent : il est rattaché à l'animal, et plus précisément à la vie comme survie, mais il ouvre aussi l'homme au registre du «transanimal » c'est-à-dire à une conception de la vie humaine fondée sur le concept d'invention plutôt que sur celui de survie et de conservation. L'outil nous situe cependant dans une différence de degré avec les autres animaux, la rupture véritable (et donc la différence anthropologique proprement dite) se produit avec l'image et avec le tombeau, notamment avec l'irruption des témoignages d'une activité esthétique non technique dans l'histoire humaine (Jonas évoque les dessins rupestres), ainsi qu'avec les premières manifestations d'une pensée métaphysique de l'homme comme un être voué à la mort.

Sans faire à Jonas un procès malvenu en lui opposant des savoirs actuels qui démentent sur un certain nombre de points les assertions qu'il pouvait soutenir dans les années 1980-1990 (comme par exemple le fait que l'outil soit le propre de l'homme: il semble aujourd'hui que les premiers tailleurs de silex doivent plutôt se recruter parmi les Australopithèques), il convient d'évaluer la construction jonassienne au regard des savoirs paléoanthropologiques sur lesquels il pouvait effectivement s'appuyer à l'époque où il a rédigé son texte.

Il convient tout d'abord de souligner qu'en abordant l'homme par les vestiges matériels de son activité, l'outil, l'image et le tombeau, Jonas les situe dans la même profondeur de temps («j'ai opté pour les témoignages d'artefacts visibles, produits par l'homme, de ceux-là mêmes qui apparaissent très tôt et sont fort répandus dans les temps les plus reculés»). Or, si nous disposons effectivement de vestiges lithiques vieux de plusieurs millions d'années, les premières sépultures connues datent quant à elles de moins de cent mille ans, et les premières manifestations d'un art graphique de moins de cinquante mille ans. Les peintures pariétales les plus anciennes ont moins de quarante mille ans et disparaissent aux alentours du dixième millénaire avant J.-C. Sauf à considérer que l'homme dont Jonas cherche à retracer l'aventure sur terre se limite à l'homme moderne, vieux de quelques dizaines de milliers d'années, ce raccourci est donc problématique : sur la plus grande partie de son histoire, l'homme n'a laissé aucun témoignage de sa vie intellectuelle et de ses possibilités mentales autre que les vestiges de ses industries lithiques. Nous n'avons aucune sépulture, aucune image d'Homo habilis. En outre, en acceptant malgré tout de considérer l'homme moderne seulement, un fait troublant est complètement passé sous silence par Jonas: les peintures pariétales, témoignages par excellence d'une rupture d'essence avec l'animalité (selon Jonas), ont une distribution géographique très limitée, bien plus étroite que l'occupation humaine de la Terre. On les retrouve essentiellement dans ce que l'on appelle le franco-cantabrique, c'est-à-dire dans le sud-ouest de la France et au nord de l'Espagne. Il y a des sites ailleurs, mais très peu nombreux et surtout, des continents entiers sont pour ainsi dire vides de témoignages l'Asie, l'Afrique, l'Amérique. Faut-il alors considérer que seuls les hommes du francocantabrique, c'est-à-dire les seuls ou presque à avoir laissé des témoignages aussi impressionnants de la faculté à fabriquer des images, sont les seuls hommes véritables? Faut-il conclure que toutes les espèces humaines qui se sont succédé sur la surface de la terre depuis Homo habilis, il y a environ deux millions et demi d'années, ne méritent pas 
complètement le nom d'homme faute d'avoir laissé des témoignages autres que ceux de leur activité technique? Autre précision: nous savons que Néanderthal enterrait ses morts et que bien sûr il taillait la pierre. En revanche, nous n'avons à ce jour aucun exemple de peinture pariétale néanderthalienne. Nous avons donc un Homo neanderthalensis qui est faber, qui connait le tombeau mais qui n'est pas pictor: qu'en conclure dans le système de référence de Jonas? Il conviendrait pour le moins de renverser l'ordre pour lequel Jonas dit « opter » : le processus ne conduirait pas de l'outil à la sépulture en passant par l'image, mais de l'outil à l'image en passant par la sépulture - s'il faut bien sûr absolument conserver cette vision d'une ascension linéaire vers l'esprit à partir des différents types de témoignages laissés par nos ancêtres, ce qui est aux antipodes de la démarche des préhistoriens aujourd'hui (même si cette vision linéaire et progressiste de l'humanité a pollué la préhistoire pendant plusieurs décennies).

La paléoanthropologie et la préhistoire contemporaines nous confrontent au défi de penser l'aventure humaine à travers la diversité des espèces humaines, des formes d'humanité qui ont peuplé la terre et des témoignages lacunaires de leur vie matérielle, sociale et mentale. Le constat que plusieurs formes d'humanité se sont succédées sur une période de plus de deux millions d'années interdit de considérer comme une donnée de départ l'unité de l'humanité : cette dernière ne peut pas être postulée, elle est une question, peut-être une tâche à accomplir. Dans ces conditions, quelle signification l'idée d'une « image de l'homme " peut-elle recevoir? Peut-on encore parler d'une « image de l'homme »? Cette idée n'est-elle pas pour ainsi dire en contradiction avec l'affirmation principielle, étayée par la documentation accumulée, qu'il y a une diversité irréductible $\mathrm{du}$ « nom de l'homme»?

Le traitement que Jonas fait des tombeaux illustre bien cette déconnexion relative entre la construction philosophique et les savoirs scientifiques. En effet, Jonas voit dans la sépulture la manifestation par excellence du «transanimal », c'est-à-dire d'une existence vouée à la vie de l'esprit. L'histoire de la préhistoire confirme en un sens le point de vue de Jonas : en effet, l'étude des pratiques funéraires préhistoriques a été le dernier bastion de la préhistoire la moins scientifique, livrée aux «fouilleurs » amateurs opérant sans critique et sans méthode, organisant et même trafiquant ${ }^{20}$ les matériaux en vue d'étayer une préconception de l'homme comme un être avant tout spirituel. Toutefois, la situation change dans les années 1960, en particulier avec la fouille de la sépulture préhistorique des Mournouards par Leroi-Gourhan et son équipe en 1966 : Leroi-Gourhan jette les bases d'une nouvelle discipline préhistorique, fondée sur l'étude méthodique des objets déposés dans les tombes, de la position des corps, du nombre d'individus présents dans chaque sépulture etc. Leroi-Gourhan insiste lourdement sur un point : il est question de décrire des pratiques, des gestes opératoires, mais certainement pas de remonter de là aux « rites » ou aux « croyances » des Paléolithiques. Dans le domaine de l'art pariétal, auquel Leroi-Gourhan a consacré plus de vingt ans de son activité de recherche (ainsi que son enseignement au Collège de France), il en va de même: les grottes ornées témoignent d'une pensée hautement élaborée, sans doute de l'existence d'un Paléolithique métaphysicien, mais le contenu de ces représentations nous est à jamais perdu. Nous avons accès à la syntaxe, mais pas aux significations au sujet desquelles seules des conjectures sont permises.

32 Sans même parler de Leroi-Gourhan, que Jonas n'avait sans doute pas $l^{21}$, il est possible de mentionner un autre savant auteur d'une théorie originale de l'hominisation, parue en 1970 , que Jonas aurait très bien pu lire. Cet ouvrage ${ }^{22}$ a connu un franc succès et a été très 
discuté à l'époque, l'absence de confrontation entre la conceptualité jonassienne du «transanimal» et la documentation paléoanthropologique ne s'explique donc pas seulement par une quelconque difficulté d'accès à celle-ci. Cet auteur, Paul Alsberg, était médecin et anthropologue. Sa conception originale de l'hominisation a consisté à renverser complètement le point de vue communément admis d'un homme nu, sans ressource, obligé de s'outiller pour survivre. En effet, selon Alsberg, l'homme est initialement un Primate parfaitement adapté à son environnement, utilisant à l'occasion des objets en guise d'outils, comme le font d'autres animaux. La faculté d'utiliser des objets comme outils se perd donc dans l'animalité, puis des hasards vont mettre l'homme en présence d'éclats de silex de plus grande efficacité, ce qui l'aurait amené à fabriquer intentionnellement ces éclats. L'utilisation plus systématique d'outils aurait alors entraîné une régression progressive de l'équipement naturel et fait apparaître l'homme comme le vivant démuni. En réalité, c'est le mouvement inverse qui s'est produit: l'homme ne s'est pas outillé pour compenser une nudité constitutive, il s'est "débarrassé » de ses attributs dès lors qu'il a adopté un mode de confrontation à la matière fondé sur la fabrication d'artefacts extériorisés. Au processus de body-adaptation aurait succédé un processus de body-liberation. La théorie de Alsberg n'est pas celle de Leroi-Gourhan, mais les deux auteurs insistent sur l'idée que l'outil a un ancrage vital, et qu'il est un élément décisif dans le mouvement par lequel la vie s'est faite vie humaine. Le moteur de l'évolution vers l'homme n'a pas été l'intelligence ou l'esprit, mais le fait que l'évolution de l'homme en tant qu'être vivant s'est faite par des artefacts extérieurs à l'organisme.

Il n'y a sans doute pas lieu ici d'opposer la vision scientifique supposée exacte de l'hominisation (pas plus Alsberg que Leroi-Gourhan ne sont parole d'Evangile pour les préhistoriens d'aujourd'hui), à une vision philosophique qui serait suspecte par principe il est toutefois permis de demander des comptes à Jonas pour son silence concernant les savoirs de la paléoanthropologie, de la préhistoire et de l'éthologie, à une époque où il n'est pas (plus?) défendable de proposer une anthropologie qui à aucun moment n'entre en discussion avec la documentation scientifique. On peut d'autant plus regretter ce qui apparait comme un rendez-vous raté, que la philosophie jonassienne fournit de façon remarquable un cadre d'interprétation des techniques du point de vue de la vie - ce qui ouvre indéniablement sur des perspectives très stimulantes et fructueuses aujourd'hui, dans le contexte des technologies dites "convergentes" (biotechnologies, nanotechnologies). Celles-ci sont en effet le plus souvent traitées dans la littérature d'un point de vue de philosophie morale et politique, ou bien d'un point de vue épistémologique, mais très rarement d'un point de vue qui considère la technique comme un fait inscrit dans la vie et dans son mouvement. On a donné plus haut quelques aperçus de la fécondité de la pensée de Jonas pour aborder de façon originale, à partir de la vie, ces nouveaux domaines de la recherche et de l'innovation technologiques à l'échelle moléculaire. La possibilité aujourd'hui d'engager la philosophie jonassienne de la technique dans les problèmes les plus épineux posés par les technologies convergentes, au-delà des célèbres analyses du début du Principe responsabilité, risque cependant de perdre en puissance, sauf à ce que cette philosophie ambitieuse et stimulante de la technique soit découplée d'un projet d'anthropologie philosophique qui reste peu informé des connaissances scientifiques concernant le statut biologique de la technique, chez l'homme et chez l'animal. 


\section{NOTES}

1. H. Jonas, Essais philosophiques. Du credo ancien à l'homme technologique (1980), Paris, Vrin, 2013.

2. Ibid., p. 17.

3. Sous-titre de Le principe responsabilité.

4. H. Jonas, Le phénomène de la vie. Vers une biologie philosophique (1966), Bruxelles, Editions De Boeck Université, 2001.

5. A. Leroi-Gourhan, Le Geste et la parole, deux tomes, Paris, Albin Michel, 1964 et 1965.

6. Voir par ex. H. Jonas, L'Art médical et la responsabilité humaine (1985), Paris, Les Editions du Cerf, 2012, pp. 23-32.

7. Ibid., p. 37.

8. H. Jonas, Le phénomène de la vie.

9. Ibid., p. 23.

10. Ibid., p. 27.

11. Ibid., p. 20.

12. H. Jonas, Une éthique pour la nature, Paris, Desclée de Brouwer, 2000, p. 145.

13. H. Jonas, Le phénomène de la vie. Vers une biologie philosophique, op. cit., p. 40.

14. Idem.

15. Idem.

16. H. Jonas, « Outil, image et tombeau. Du transanimal dans l'humain », in Évolution et liberté (1992), Paris, Bibliothèque Rivages, 2000, p. 76.

17. Ibid., pp. 59-82.

18. Ibid., pp. 62-63.

19. H. Jonas, « Outil, image et tombeau. Du transanimal dans l'humain », op. cit.

20. Il y a eu quelques cas de fraudes avérées.

21. La traduction anglaise de Le Geste et la parole paraît en 1993, soit après la parution du texte commenté ici.

22. P. Alsberg, In Quest of Man. A Biological Approach to the Problem of Man's Place in Nature, Oxford, Pergamon Press, 1970. 